\title{
Optimizing the Sandwich Composite Structure in the Cantilever Beam
}

\author{
Tuo Hu \\ Shenzhen Foreign Languages School, Shenzhen, China \\ Email: richardhut@126.com
}

How to cite this paper: $\mathrm{Hu}, \mathrm{T}$. (2017) Optimizing the Sandwich Composite Structure in the Cantilever Beam. Modern Mechanical Engineering, 7, 127-143. https://doi.org/10.4236/mme.2017.74009

Received: August 25, 2017

Accepted: November 14, 2017

Published: November 20, 2017

Copyright (C) 2017 by author and Scientific Research Publishing Inc. This work is licensed under the Creative Commons Attribution International License (CC BY 4.0).

http://creativecommons.org/licenses/by/4.0/

\begin{abstract}
The sandwich structure is of great interest because of its advantage of combining light weight and high flexural stiffness. Many previous researchers have studied the failure modes in sandwich structures and the effects on the load capacity caused by the change of the constituent materials' properties. In this research, by applying Finite Element Analysis (FEA) method, we simulated a cantilever beam composed of a sandwich structure in Abaqus, to find out the preferred design principles that help decrease the stress and displacement in the beam when applied a uniform load. We also determined the effect of the core geometry on decreasing the displacement and the stress in the beam.
\end{abstract}

\section{Keywords}

Cantilever Beam, Sandwich Structure, Stress, Free End Displacement

\section{Introduction}

A Sandwich structure is normally composed of two thin outer layers (faces) with a large density and a thick inner layer (core) with a smaller density. Compared with traditional beams that consist of a single material, the sandwich panel is being more and more widely used in real applications because it can significantly reduce the beam weight while maintaining an adequate flexural stiffness. Decreasing the weight can reduce the possibility of the catastrophic failure caused by fatigue. And there are other factors that could lead to the failure. Under high pressure, once the axial stress on the faces exceeds the tensile stress of the constituent material, the structure would experience plastic strain and finally fail, so does the core. The failure modes in the composite structures have been extensively investigated through experimental or numerical methods [1]-[9]. A general review of failure modes in sandwich structures was presented by Broughton 


\section{[2], Allen [10] and Zenkert [11].}

Among previous published researches on sandwich structures, Miravete [12] generally discussed how the Young's Modulus and the thickness of the core and faces affect the load capacity of the sandwich panel. Gibson's [4] research was about how to minimize the weight of a sandwich panel for given structural requirements by adjusting the density and thickness of faces or the core. Recently, Saeid and Donaldson [1] studied on the effects of thickness variation of the core material on the load capacity of the structure through experiments. Their experimental results showed that the critical energy release rate could be influenced by core thickness variations. Other than the thickness studies, the geometry effects on the structure are also extensively studied [1] [3] [4] [13] [14] [15]. For example, Konsta [3] focused on how the geometry of the core can affect the failure mode by comparing foam and honeycomb core design.

In this present work, we summarized the effect of Young's modulus and thickness variation on the load capacity of the structure by simulation analysis with FEA method. The advantage of the simulation compared to previous experimental or numerical study is that we can clearly see the stress and displacement distribution in the beam when modifying the sandwich structure. The simulation also tells us that varying thickness is better in terms of load capacity compared to changing Young's modulus.

Furthermore, we studied the effect of the core geometry on the stress and the displacement distribution. The focus of the study is on changing the hollow structure of the core and seeing its effect on the load capacity. What we found is that the side area of the hollow structures is one important factor that affects the load capacity. And this gives us guidance on the design of hollow core structures in sandwich beams in the future.

\section{Simulation Analysis}

The simulation was run using FEA method in Abaqus 6.14. The details are described in the following sections.

\subsection{Material Properties}

In our simulation, two materials are employed in the models: steel and polystyrene (PS). The details of these materials are shown in the Table 1.

The property of the steel here is from ASTM A36 carbon steel [5] and that of polystyrene is found from Bangs Laboratories Inc. [6].

Table 1. The properties of steel and polystyrene.

\begin{tabular}{ccc}
\hline & Steel & Polystyrene \\
\hline Density $\left(\mathrm{g} / \mathrm{cm}^{3}\right)$ & 7850 & 1040 \\
Young's Modulus (GPa) & 209 & 3 \\
Poisson's Ratio & 0.3 & 0.3 \\
\hline
\end{tabular}


Steel has a higher stiffness, PS can help with the load distribution and reduce the weight of the overall sandwich structure. In our sandwich structures, steel is used as the face materials as the core material.

\subsection{Abaqus Models}

To determine which element type to use, we built a cantilever beam with a rectangular cross section. The details of this model are shown in Figure 1. The three dimensions of the beam are: $400 \mathrm{~mm}, 100 \mathrm{~mm}$, and $50 \mathrm{~mm}$. Two boundary conditions are applied here. First, one end is entirely fixed on the wall while the other one is free. On the other hand, a pressure of $0.4 \mathrm{Mpa}$ is applied uniformly on the top of the beam as the loading step. These two boundary conditions are applied in the following simulations.

When building a sandwich structure in Section 3.3, we made the thickness of the face and the core equal, as shown in Figure 2(a). In Section 3.4, we used beams with dimensions shown in Figure 2(b) and Figure 2(c). These two figures show the change in the thickness of the core and the faces respectively. The model shown in Figure 2(b) is also applied in Section 3.5.

\subsection{Mesh Density and Element Type}

In all models, we used C3D20 as our element type with a mesh density of 0.01 . The reason of choosing C3D20 will be elaborated in the following section.

\section{Results and Discussion}

\subsection{Element Type Selection}

To find out which element type works the best, we tried C3D4, C3D8 and C3D20 in the model of all steel cantilever beam and compared the free end displacement with analytical results. Here, the analytical vertical displacement $y$ is determined by the equation below:

$$
y=\frac{w x^{2}\left(x^{2}+6 l^{2}-4 l x\right)}{24 E I}, I=\frac{b h^{3}}{12}
$$

where $w$ is known as the distributed load, $E$ is the Young's modulus, $l$ is the length of the beam, $b$ is the dimension parallel to the bending axis, and $h$ is the

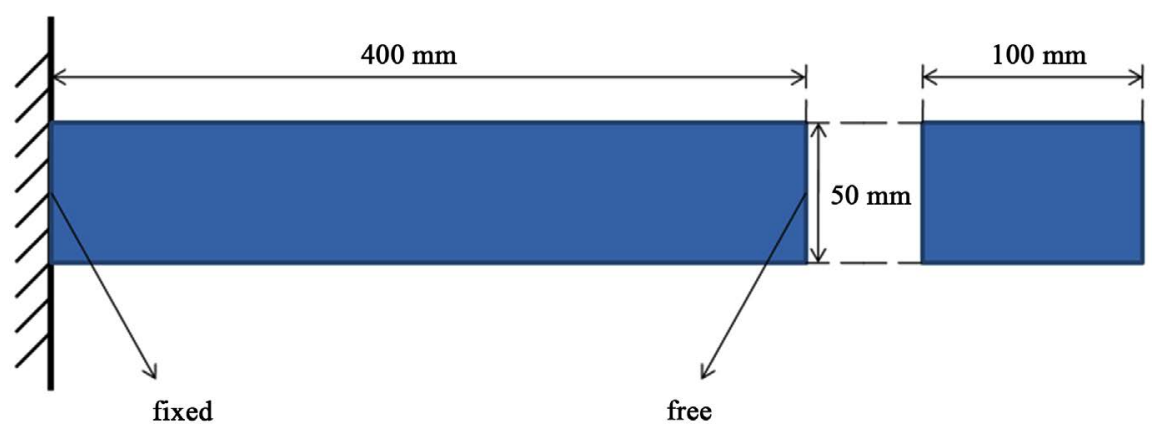

Figure 1. The beam cross section for Section 3.1. 


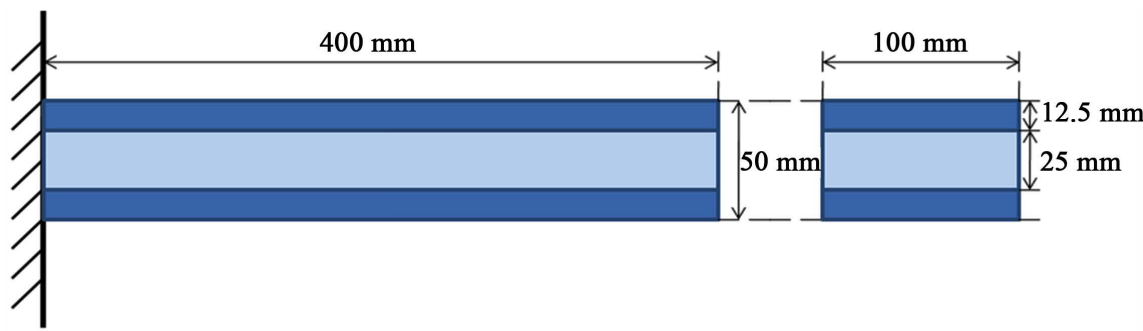

(a)

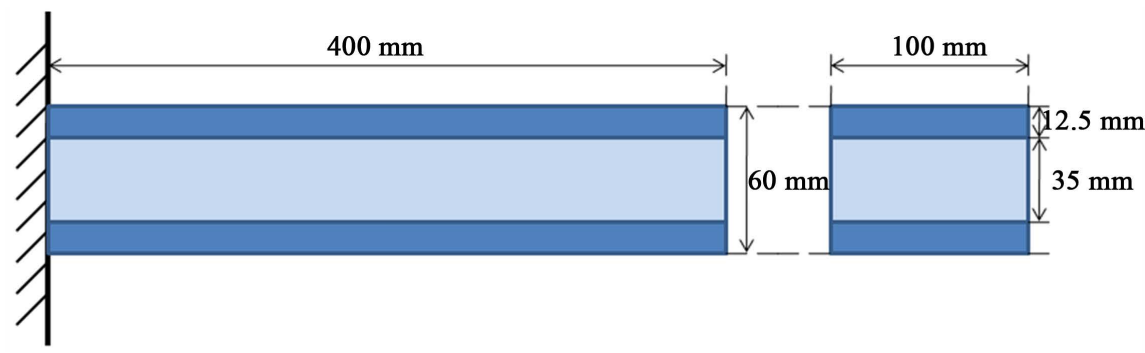

(b)

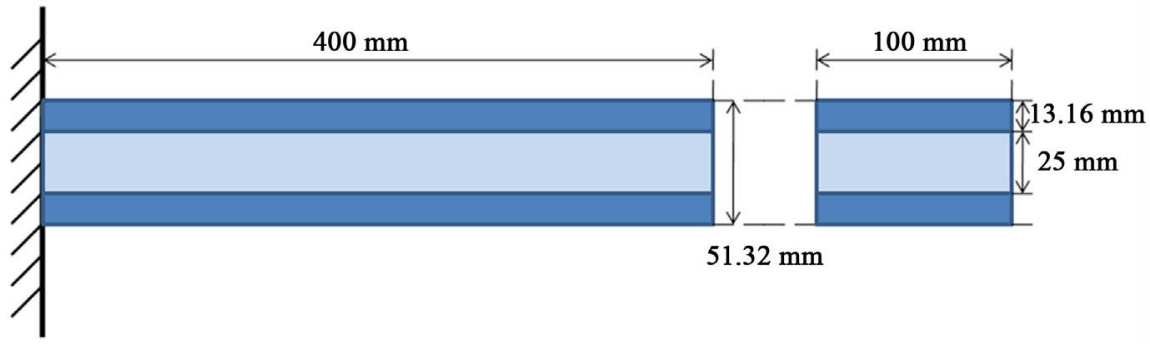

(c)

Figure 2. The beam dimensions in different models. (a) The model in Figures 4-6, The model in Sections 3.2 \& 3.3 (changing stiffness); (b) the model in Figure 7(a) \& Figure 7 (c), and Figure 9 \& Figure 10, the model in Section 3.3 (changing the thickness of core) \& 3.4; (c) the model the model in Figure 7(b) \& Figure 7(d), the model in section 3.3 (changing the thickness of faces).

dimension perpendicular to the axis. In our condition, $l=0.4 \mathrm{~m}, b=0.1 \mathrm{~m}$, $h=0.05 \mathrm{~m}, p=0.4 \mathrm{MPa}$ and $w=p * b=0.04 \mathrm{MN} / \mathrm{m}$.

The comparison between the simulated and analytical vertical displacement solution is shown in Figure 3. The vertical displacement is the amount of displacement in the direction of the force, and the $\mathrm{X}$ distance is the distance from a point to the fixed wall in the direction parallel to the beam length.

It can be seen that the result using C3D4 element type deviates the most from the analytical result, while either C3D8 or C3D20 gives a more accurate displacement. Table 2 shows the error of the free end displacement derived from the simulation is within $1 \%$. To maintain the low error in the following simulation, we used C3D20 in subsequent models.

\subsection{The Advantage of a Sandwich Composite Structure}

To convince that sandwich structures are more applicable in construction than all steel beams, we compared the stress and displacement of these two structures 


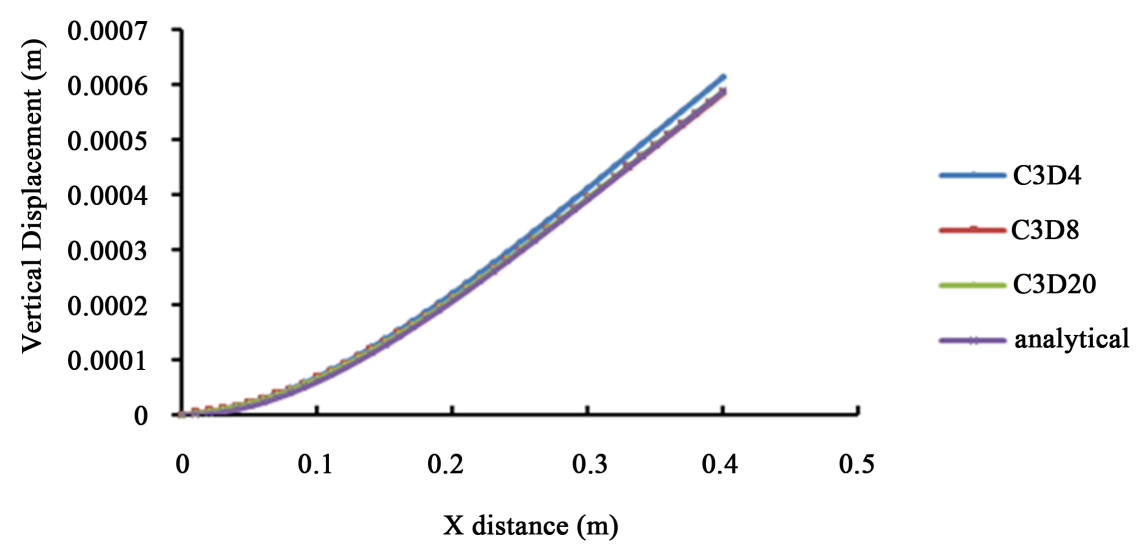

Figure 3. The comparison of the vertical displacements among different element types.

Table 2. The comparison between the vertical free end displacements derived from C3D8 and $\mathrm{C} 3 \mathrm{D} 20$.

\begin{tabular}{ccc}
\hline Element Type & Vertical Free End Displacement Magnitude $(\mathrm{m})$ & Error (\%) \\
\hline C3D8 & $5.85 \mathrm{E}-04$ & 0.44 \\
C3D20 & $5.88 \mathrm{E}-04$ & 0.08 \\
Analytical & $5.88 \mathrm{E}-04$ & \\
\hline
\end{tabular}

under the same amount of load. In the simulation, we used the single variable method: the only difference is the change of the inner material of the beam. The parameters of the panels are shown respectively in Figure 1 and Figure 2(a).

From the graph shown above, the free-end displacement of the all steel beam is $44.30 \%$ smaller than that of the composite panel; the upmost stress of the all steel beam is $42.49 \%$ smaller than that of the composite panel. However, according to the data of both materials listed in Section 2.1, the density of steel is more than six times larger than that of the sandwich composite beam. The heavy burden caused by the weight of the all steel beam far exceeds the advantage brought by its smaller displacement and stress. Also, according to Figure 4(d), the maximum stress on PS is $12.08 \mathrm{MPa}$, which is only $40.27 \%$ of the tensile stress of PS ( $\sim 30 \mathrm{MPa})$; the maximum stress on steel is $144.3 \mathrm{MPa}$, which is only $49.76 \%$ of the tensile stress of steel ( $290 \mathrm{Mpa})$. Therefore, adding a layer of PS core would not lead to catastrophic failure. As a result, we prefer to choose the sandwich structure in practice for its lighter weight.

\subsection{The Role of Faces and the Core in the Sandwich Structure}

In the design of sandwich structures, people use two materials with different densities and stiffnesses. To determine whether the stiffer material should be employed as the core or the faces, we simulated a composite composed of steel and PS. The model used here is shown in Figure 2(a). The details of the simulation are descrived in the simulation analysis section.

Figure 5(a) and Figure 5(b), show the displacements in (1) Steel as the face material; (2) PS as the face material. The displacement of model (1) ranges from $0 \mathrm{~m}$ 


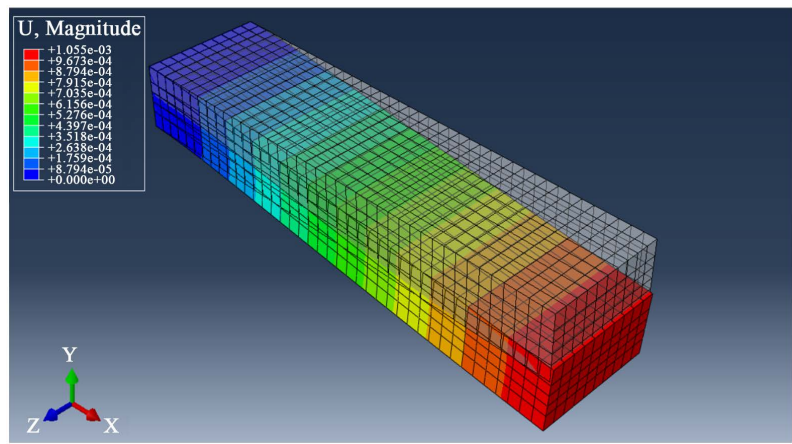

(a)

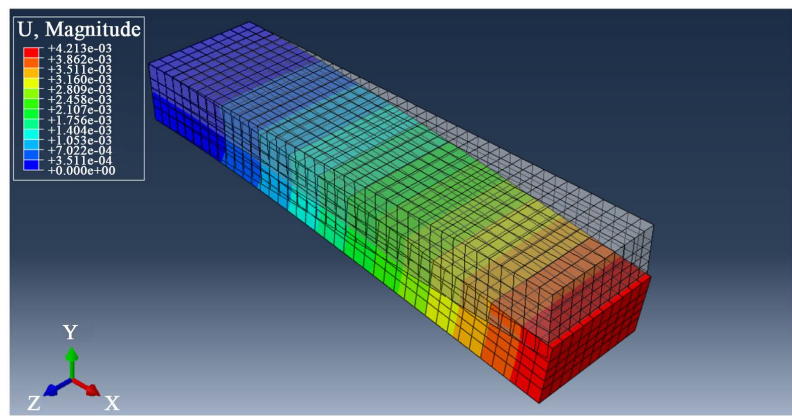

(b)

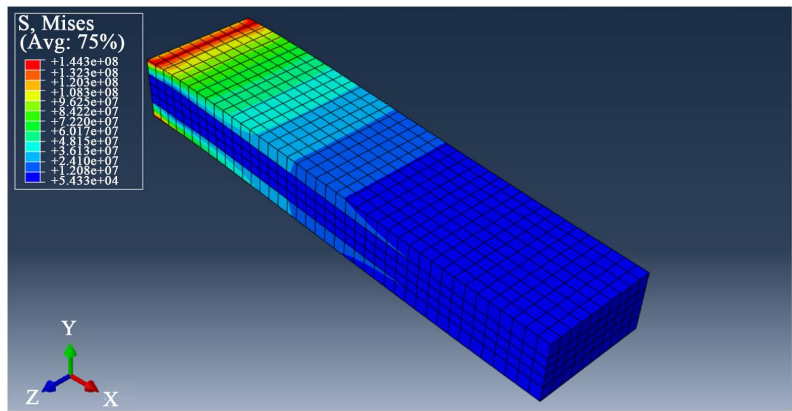

(c)

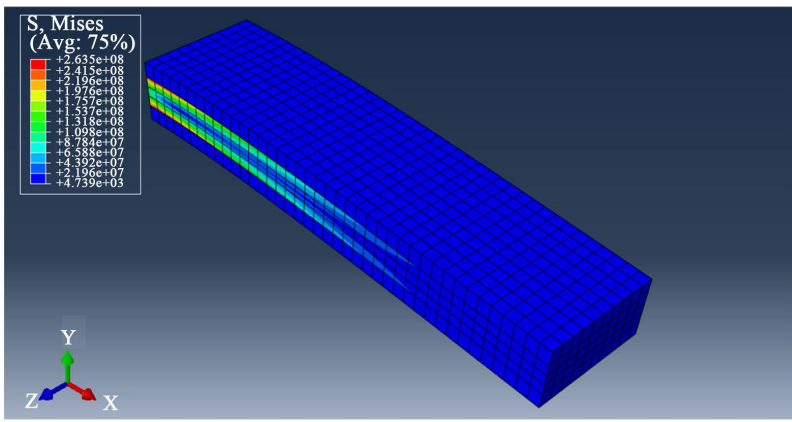

(d)

Figure 4. The comparison of the displacement and stress distribution between the all steel beam and the composite beam (the gray part shown in the displacement graphs are the original beam position). (a) Displacement of the all steel beam, The displacement when Fe is used as the face material; (b) displacement of the composite beam, The displacement when PS is used as the face material; (c) stress distribution of the all steel beam, The von mises stresses when steel is used as the face material; (d) stress distribution of the composite beam, The von mises stresses when PS is used as the face material. 


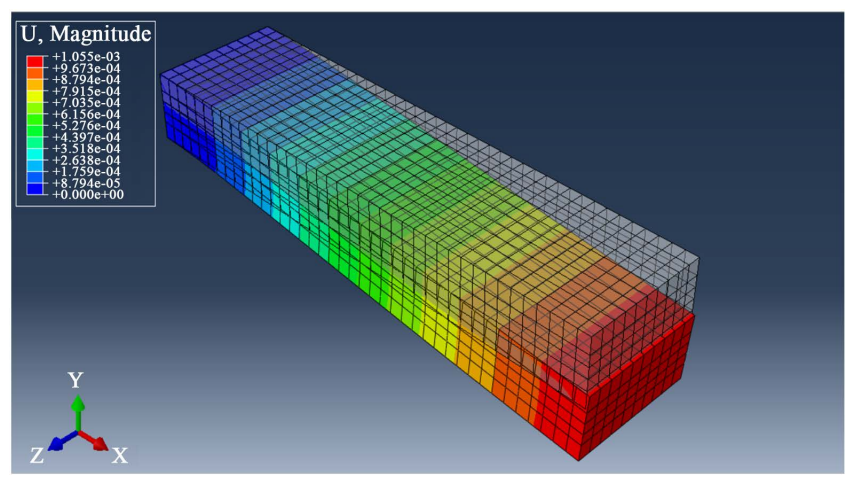

(a)

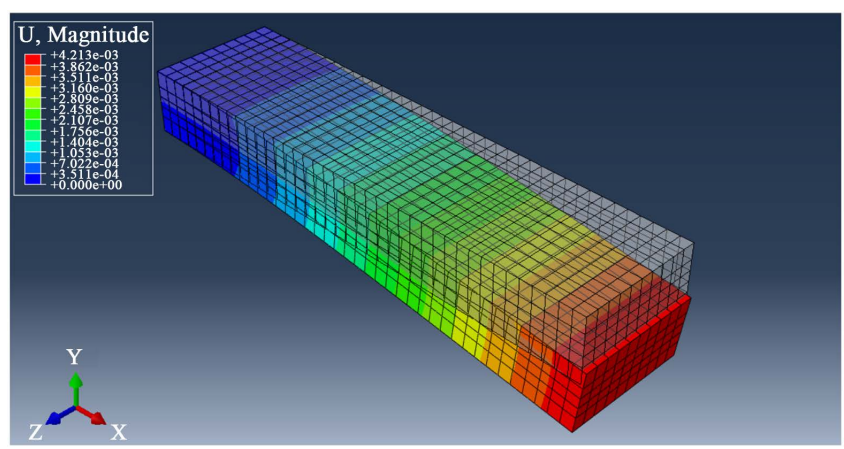

(b)

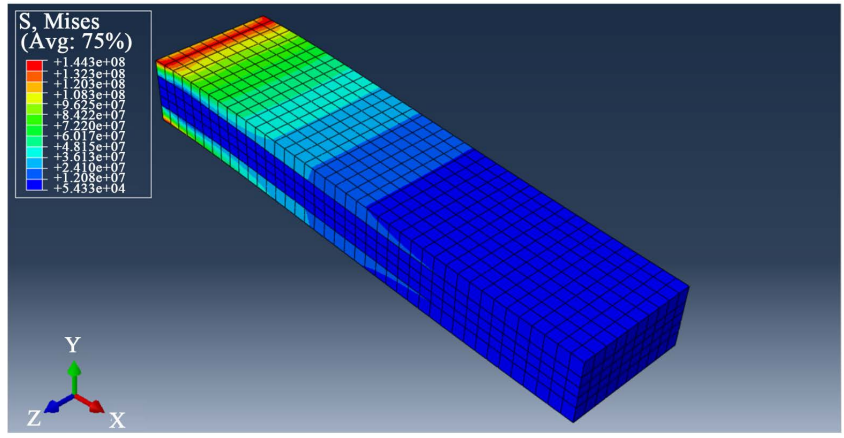

(c)

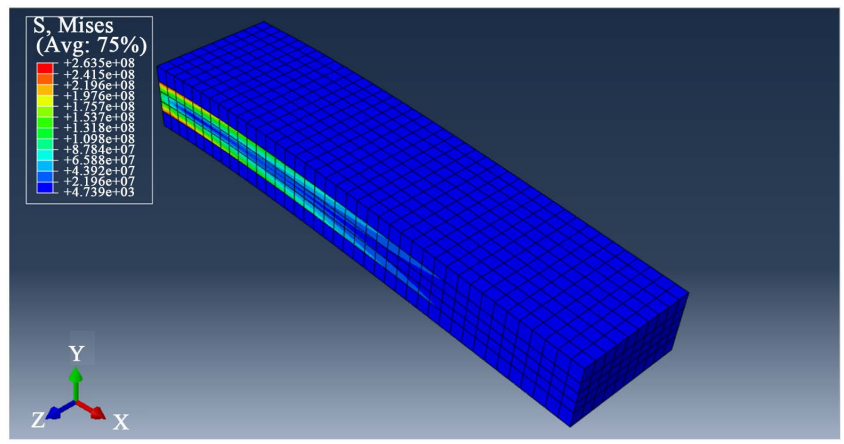

(d)

Figure 5. The displacement and stress distribution in the sandwich structure (the gray part shown in the displacement graphs are the original beam position). (a) The displacement when steel is used as the face material; (b) the displacement when PS is used as the face material; (c) the von mises stresses when steel is used as the face material; (d) the von mises stresses when PS is used as the face material. 
to $1 \mathrm{E}-03 \mathrm{~m}$ while that of model (2) goe sup to $4 \mathrm{E}-03 \mathrm{~m}$, about three times larger. For the maximum stress, the one in model (2) is about 2 times of that in model (1). This indicates that having the stiffer material as faces is a better choice in terms of smaller displacement and stress. From Figure 5(c), it can be seen the stress in the faces are much larger than that in the core which further indicates that the faces carry most of the axial stress, therefore, are a predominant determinant of the overall bending strain in the composite structure. The core, on the other hand, acts more as a buffer layer which to some extent helps carry the load. In addition, when using steel as the face materials, the stress in the faces $(144 \mathrm{MPa})$ is much smaller than the tensile strength of steel $(\sim 290$ $\mathrm{MPa}$ ) while in the other case, it's about $264 \mathrm{MPa}$. Clearly, using stiffer material as the face has an advantage. In the following study, we used steel as face materials and PS as the core material.

\subsection{Improvement on the Load and Displacement Capacity of the Beam}

The reason we chose PS as the core material is that it has a small density but a medium stiffness. This not only reduces the possibility of the failure caused by the heavy weight of the beam, but also maintains the overall stiffness of the structure. However, failure could also occur when either the axial stress on the facings or the shear stress in the core exceeds the yield stress of the constituent material. Therefore, we considered the following methods to optimize the sandwich structure:

- Increase the Young's modulus of the face material

- Increase the Young's modulus of the core material

- Increase the thickness of the face

- Increase the thickness of the core.

\subsubsection{Increasing Young's Modulus}

By increasing the Young's Modulus of the material, we meant to increase the overall stiffness of the sandwich structure. When increasing the Young's modulus of the materials, we increased it by $50 \%$ while keeping other properties the same as in Table 1. The beam dimensions are the same as in Section 3.3, and we use steel as the face material and PS as the core material. The simulation results are shown in Figure 6.

In Figure 6(a) and Figure 6(b), with increasing face stiffness, the beam experiences larger stress compared to increasing the core stiffness. When it comes to the overall displacement of the beam, it seems to be the opposite case. Detailed comparisons with the original beam are demonstrated in Table 3.

From the simulation results listed above, we can observe that the stiffer-core beam has a better affect on decreasing the stress on the beam while the stiffer-face beam has an advantage in decreasing the free-end displacement. It can be clearly seen that increasing the core stiffness is a better choice as it decreases both the stress and the displacement in the beam. 


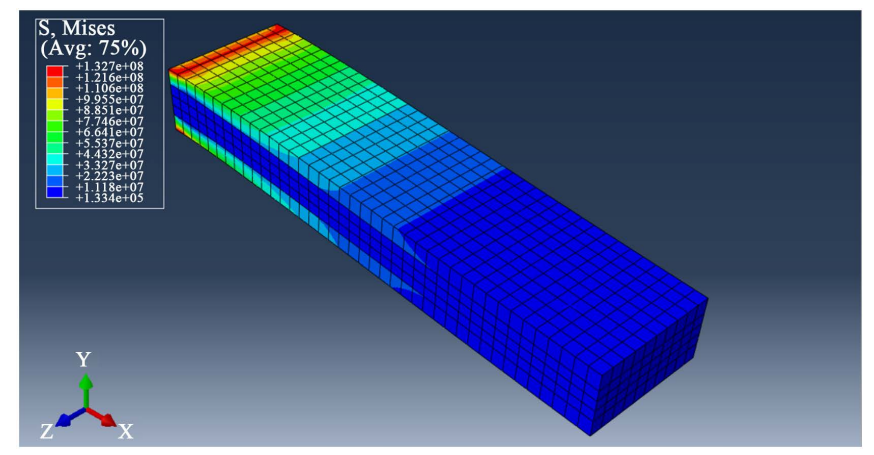

(a)

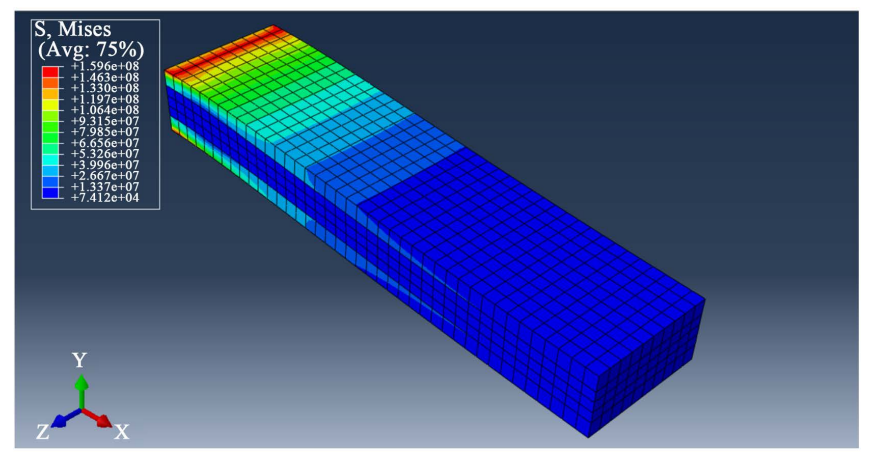

(b)

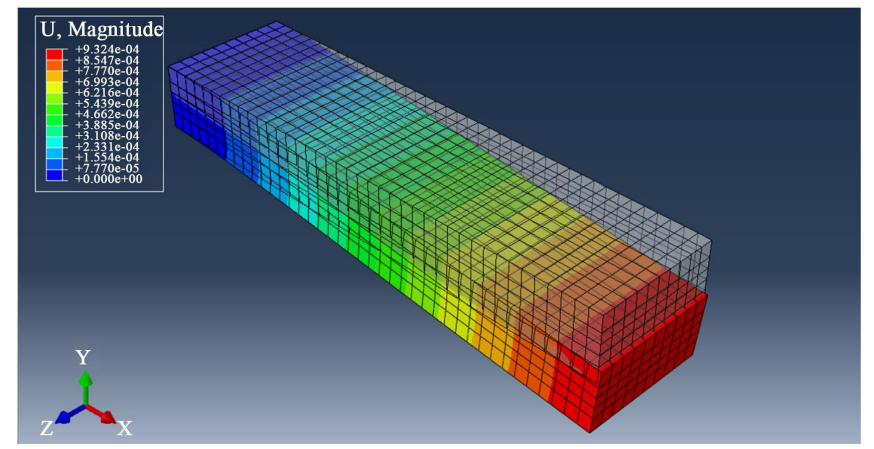

(c)

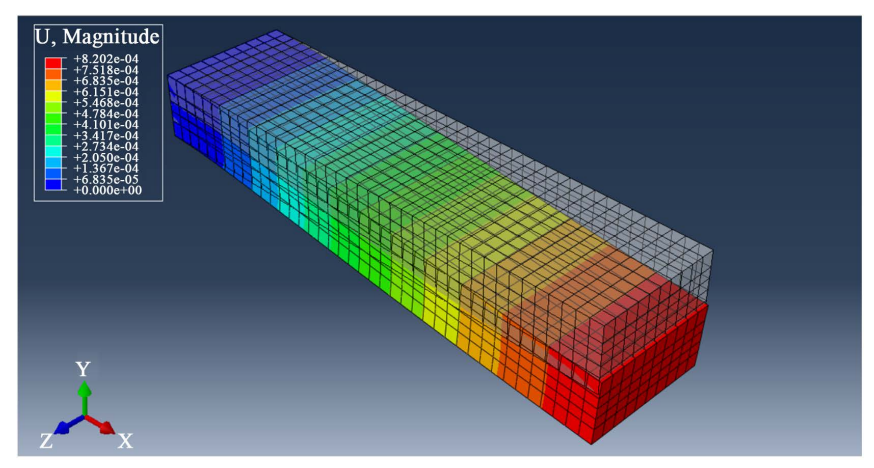

(d)

Figure 6. The displacement and stress distribution when changing the stiffness of the faces or the core (the gray part shown in the displacement graphs are the original beam position). (a) The von mises stresses when the core is stiffer; (b) the von mises stresses when the faces are stiffer; (c) the displacement when the core is stiffer; (d) the displacement when the faces are stiffer. 
Table 3. The percentage change from the original panel to the improved ones (increase the stiffness).

\begin{tabular}{cccc}
\hline Types of Structures & Change of Stress on Steel & Change of Stress on PS & Change of Displacement \\
\hline Original & $0.00 \%$ & $0.00 \%$ & $0.00 \%$ \\
Stiffer Face & $+10.60 \%$ & $+10.68 \%$ & $-22.26 \%$ \\
Stiffer Core & $-8.039 \%$ & $-7.450 \%$ & $-11.6 \%$
\end{tabular}

(+stands for increasing, and -stands for decreasing).

\subsubsection{Increasing Thickness}

On the other hand, we hope to reduce the stress in the beam through increasing the thickness of the faces or the core. We first increased the thickness of the core by $10 \mathrm{~mm}$. This adds to the overall beam mass. To make sure the mass is the same either increasing the thickness of faces or the core, we calculated the corresponding increase of the face thickness. The details of the beam dimensions are shown in Figure 2(b) \& Figure 2(c). The results are shown in Figure 7.

When we tried modifying the thickness of the beam, the improvement is much more obvious. The comparison between Figure 7(a) and Figure 7(b) shows lower stresses on both the core and the faces in the beam with a thicker core. In addition, we can observe a smaller free-end displacement of the thicker-core panel from Figure 7(c) and Figure 7(d). Detailed comparisons with the original beam are demonstrated in Table 4.

From Table 4, we can determine that thicker-core beam is a preferred choice for its larger extent of reduction in both stress and displacement.

We hypothesized a reason for this result. As a buffer layer in the panel, the PS core has an effect on spreading and mitigating the stress distribution on the beam. Increasing the thickness of the core means increasing the space where the load could be spread out, so the amount of load distributed on every point of the beam will decrease. Besides, since PS is a light material, increasing the core thickness would not lead to a significantly large burden on both steel faces. Therefore, we think increasing the thickness of the core is the preferred choice to improve the load-bearing capacity of the cantilever beam.

\subsubsection{Comparison Analysis}

Although the data seems to show that increasing the core thickness has a better scenario in reducing both the stress and the displacement than increasing the core stiffness does, we can suppose that if we enlarge the degree of increase in the core stiffness, the reduction in the stress and displacement would surpass that of the thicker-core beam shown in Table 4. In other words, since there is no standard to compare the amount of increase in stiffness and thickness, we cannot directly compare the data shown in the table to determine whether increasing the stiffness or the thickness is the better choice. However, we believe that increasing the thickness of the core is much more easily to operate in practice than changing a material's stiffness. Therefore, considering about the practicability, we chose increasing the thickness of the core as an essential method to op- 


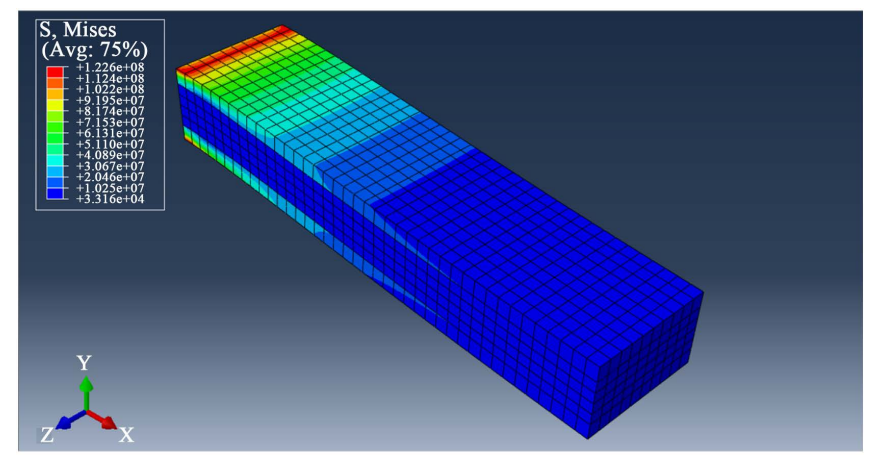

(a)

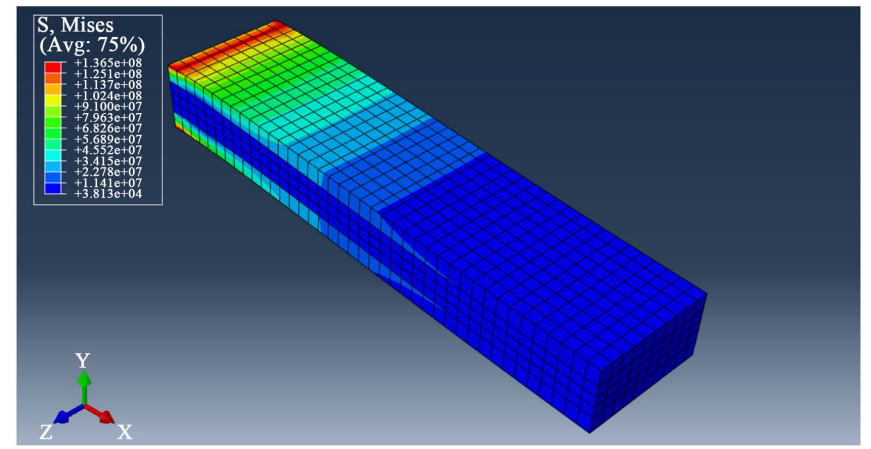

(b)

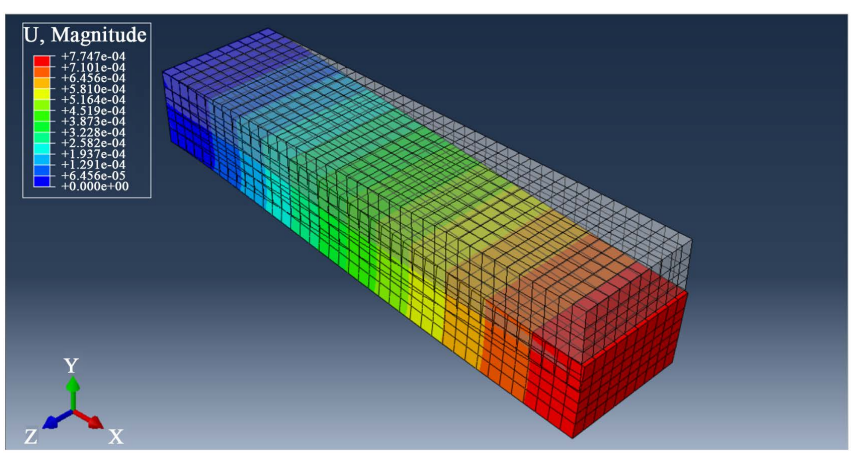

(c)

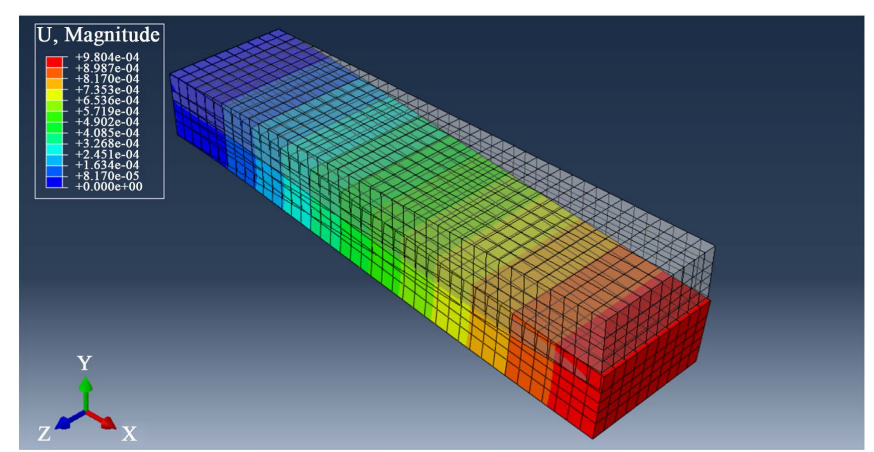

(d)

Figure 7. The displacement and stress distribution when changing the thickness of the faces or the core (the gray part shown in the displacement graphs are the original beam position). (a) The von mises stresses when the core is thicker; (b) the von mises stresses when the face is thicker; (c) the displacement when the core is thicker; (d) the displacement when the face is thicker. 
Table 4. The percentage change from the original panel to the improved ones (increase the thickness).

\begin{tabular}{cccc}
\hline Types of Structures & Change of stress on Steel & Change of stress on PS & Change of displacement \\
\hline Original & $0.00 \%$ & $0.00 \%$ & $0.00 \%$ \\
Thicker Face & $-5.405 \%$ & $-5.546 \%$ & $-7.071 \%$ \\
Thicker Core & $-15.04 \%$ & $-15.15 \%$ & $-26.57 \%$ \\
\hline
\end{tabular}

+ stands for increasing, and - stands for decreasing.

timize the design, and applied the thicker-core model (shown in Figure 2(b)) in Section 3.5 .

\subsection{How the Core Geometry Affects the Stress and the Displacement}

In order to reduce the overall beam weight, were placed the solid core with hollow structures.

This not only saves the cost, but also decreases the burden caused by the beam weight. We believe that if the contact area between the face and the core stays unchanged, the load-bearing capacity of the cantilever panel can be determined by the side surface area of the hollow parts. To confirm this, we did a series of control experiments. The designs of the hollow cores are shown in Figure 8 with an increasing side area, $\mathrm{S}$, from top to bottom: (a) $\mathrm{S}=29754 \mathrm{~mm}^{2}$, (b) $\mathrm{S}=33573$ $\mathrm{mm}^{2}$, and (c) $\mathrm{S}=37388 \mathrm{~mm}^{2}$. The hollow structures only differ in the shape of the hollow parts, other parts remain the same as in Figure 2(b). The contact area between the core and the face (the gray part in Figure 8) is the same within different models. The dimension of the panel in this section is shown in Figure 2(b). The material of the core is PS, and that the faces are made of steel.

According to the results shown in Figure 9, we can observe a decreasing trend of the maximal stresses on the beam as the side area of the hollow part increases. Judging from the displacement, although there is a slightly increase from "circles" to "squares", the overall decreasing trend of the free end displacement can still be observed. With smaller stresses or displacements, it is harder for the constituent material to exceed the yield point and fail in a catastrophic manner. Therefore, one way to optimize the hollow core parts in the sandwich structure is to increase their side areas, as shown in Figure 10.

\section{Conclusions}

The sandwich structures in our models effectively combine the high stiffness of the steel and the lightweight of the PS. Using steel as faces, they bear the most of the stress and control the panel under certain an acceptable deflection. The PS core reduces the overall weight of the beam and undertakes a certain fraction of the load.

In addition, we figured out that increasing the thickness of the core is one preferred method to optimize the design of the sandwich structure. 


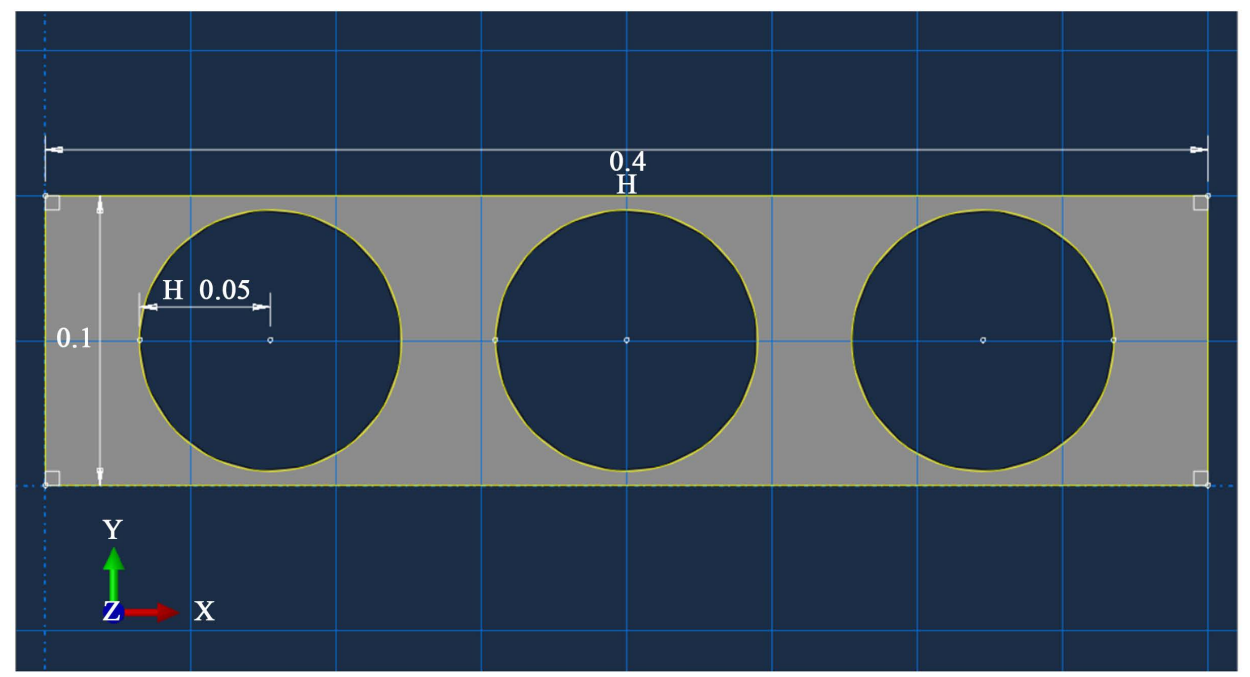

(a)

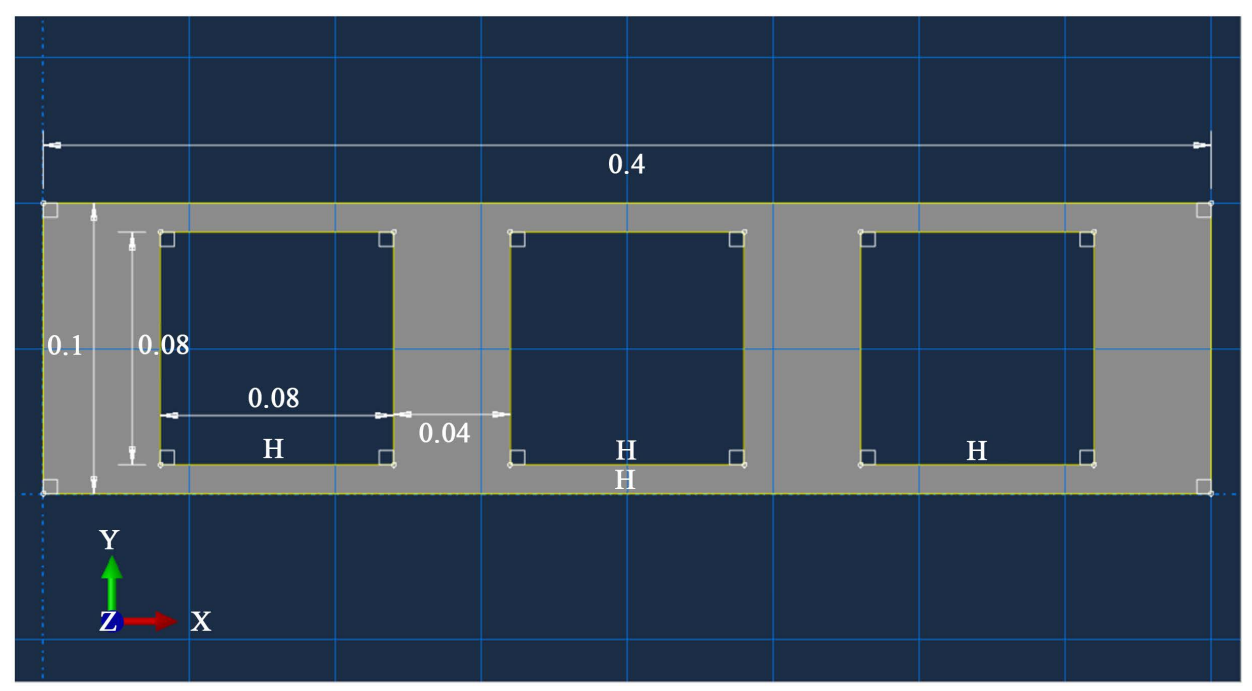

(b)

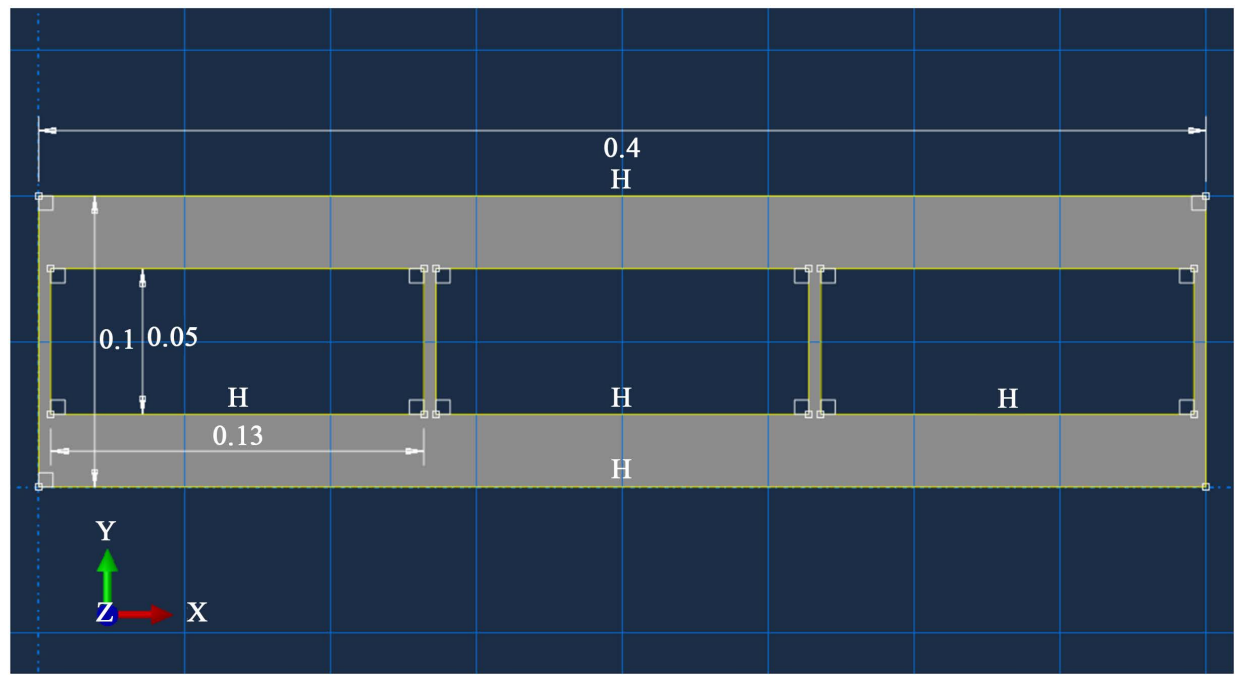

(c)

Figure 8. Cores with different hollow parts. (a) Circles; (b) squares; (c) rectangles. 


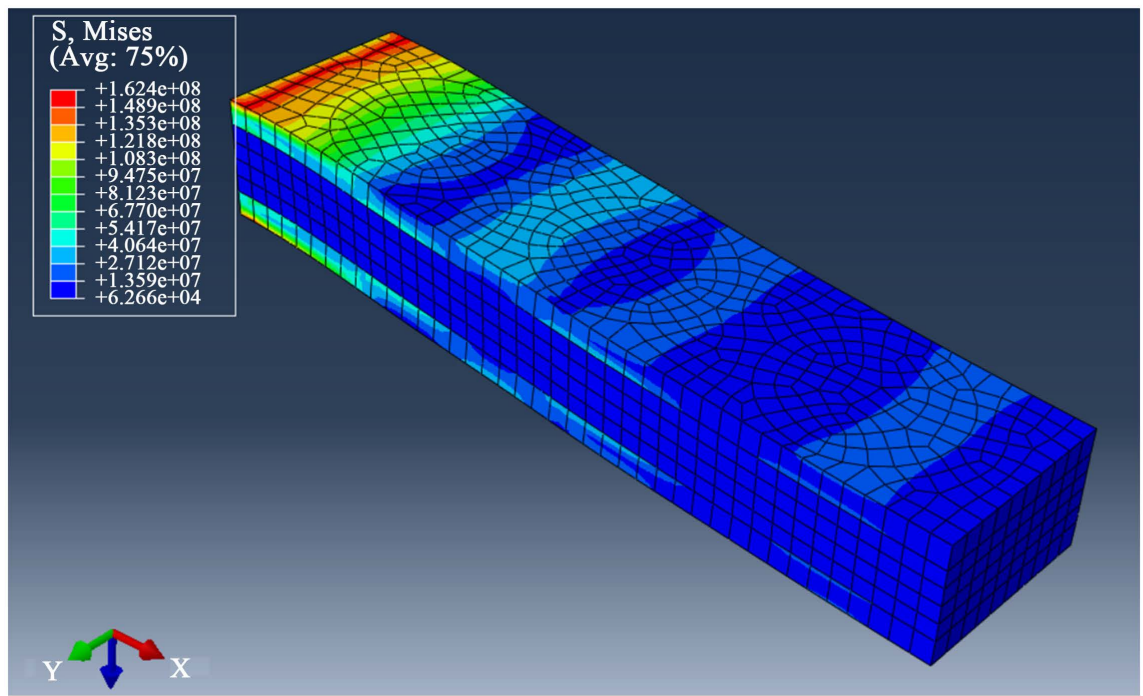

(a)

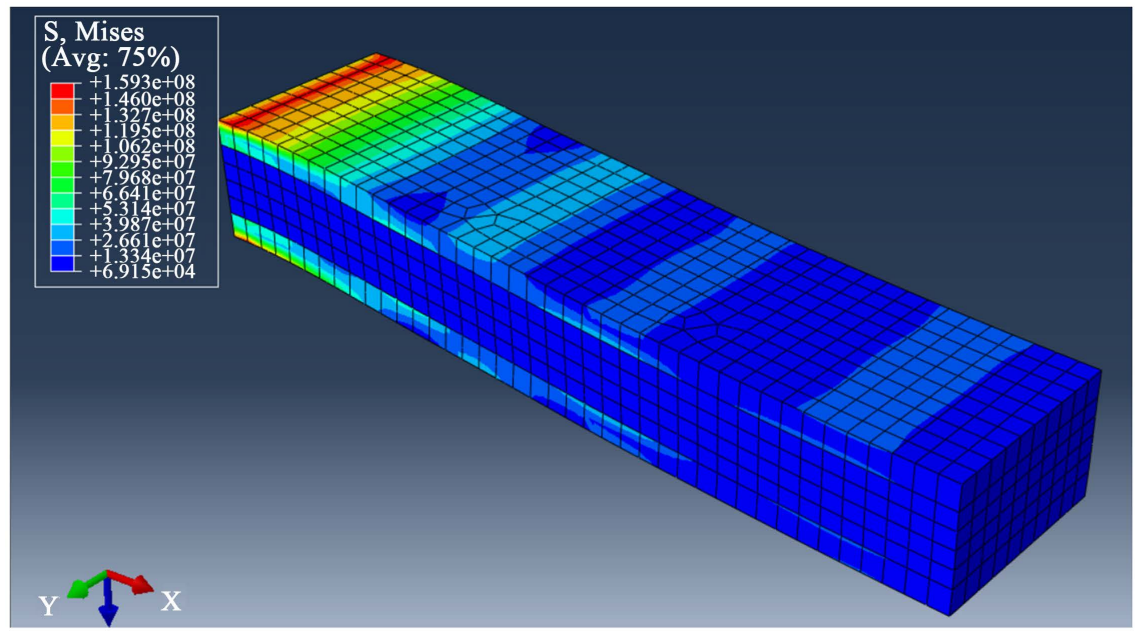

(b)

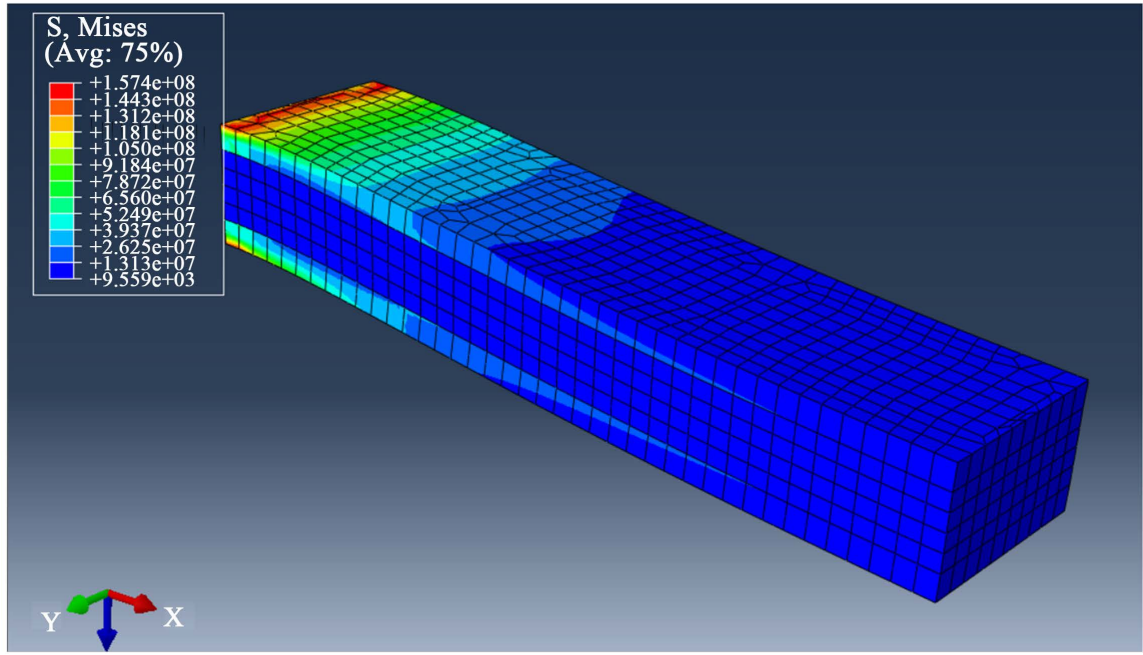

(c)

Figure 9. The von mises stresses of different core geometry. (a) Circles; (b) squares; (c) rectangles. 


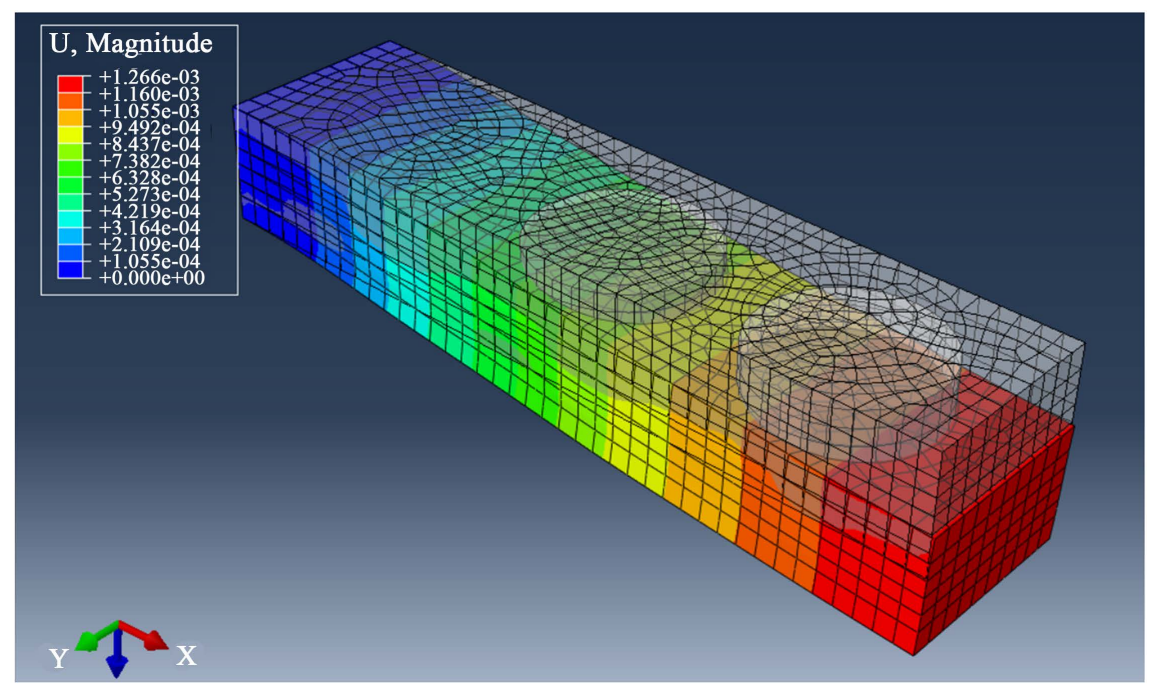

(a)

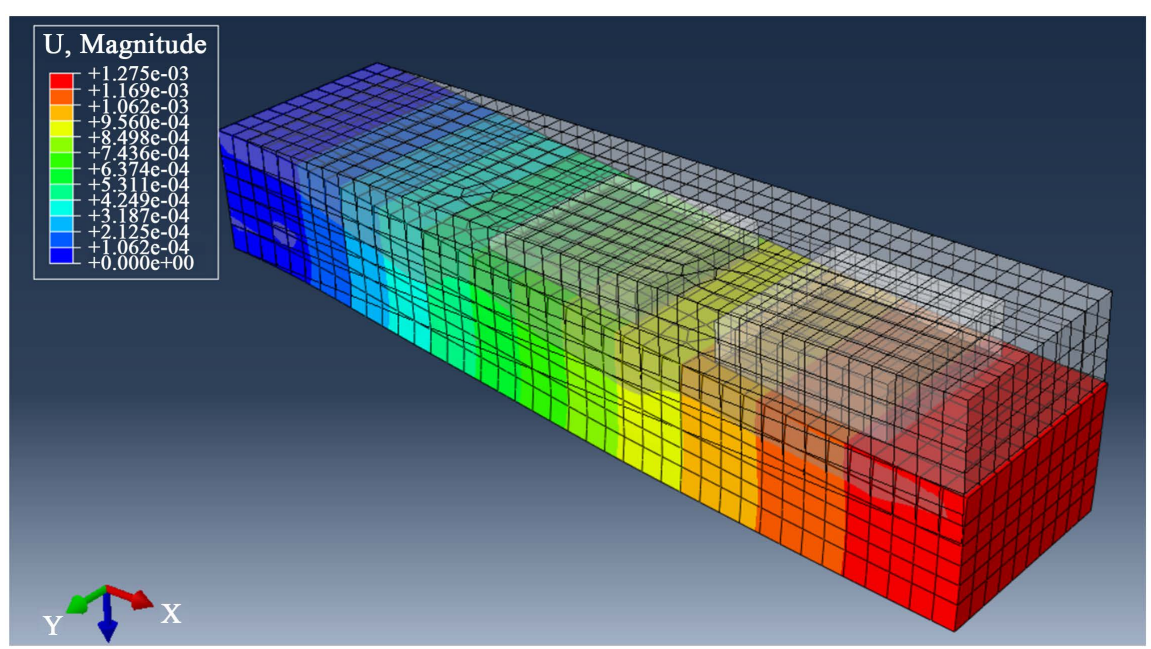

(b)

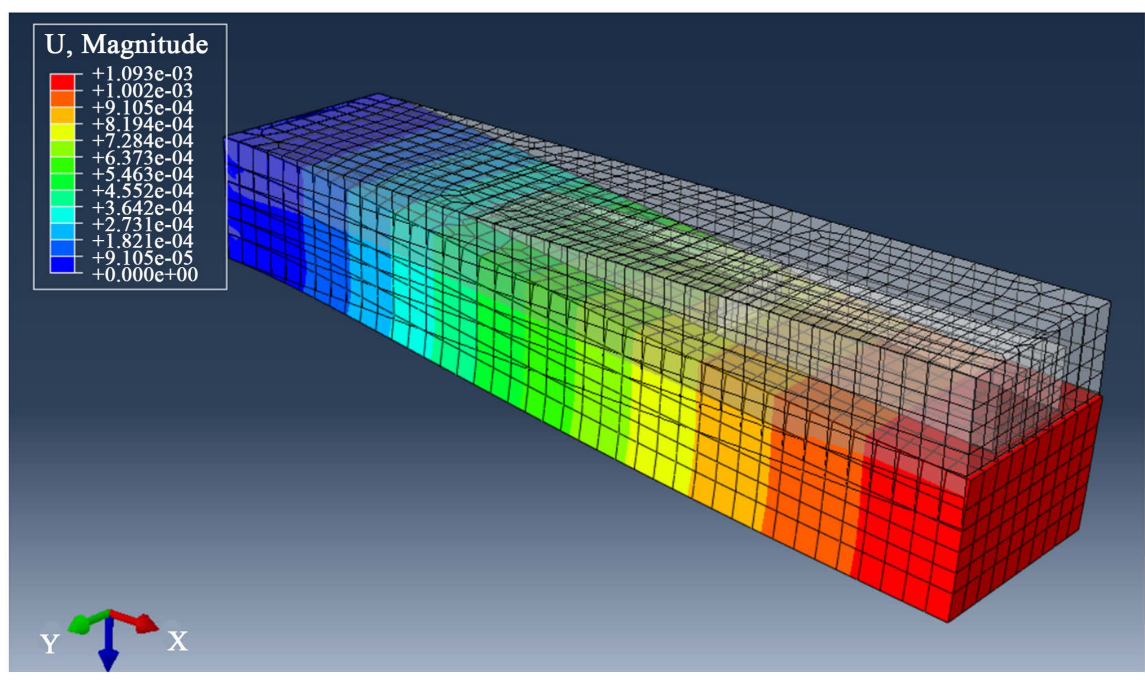

(c)

Figure 10. The displacements of different core geometry. (a) Circles; (b) squares; (c) rectangles. 
Moreover, digging hollow parts in the solid core of the sandwich structure can further decrease the weight of the beam. Surely, it will increase the overall displacement and stress. However, we find out that by increasing the side area of the hollow parts, we can reduce the maximal stress and displacement.

There are other factors, such as the geometrical distribution of the hollow parts, which can also affect the stress and displacements. More study will be carried out in the future.

\section{Acknowledgements}

This research is sponsored by Embark Education. We are also grateful to the graduate student Weizi Yuan from Northwestern University for her encouragement.

\section{References}

[1] Saeid, A.A. and Donaldson, S.L. (2016) Experimental and Finite Element Evaluations of Debonding in Composite Sandwich Structure with Core Thickness Variations. Advances in Mechanical Engineering, 8, 1-18. https://doi.org/10.1177/1687814016667418

[2] Broughton, W., Crocker, L. and Gower, M. (2002) Design Requirements for Bonded and Bolted Composite Structures. National Physical Laboratory.

[3] Konsta-Gdoutos, M.S. and Gdoutos, E.E. (2005) The Effect of Load and Geometry on the Failure Modes of Sandwich Beams. Applied Composite Materials, 12, 165. https://doi.org/10.1007/s10443-005-1120-8

[4] Gibson, L.J. (1988) Optimum Design Methods for Structural Sandwich Panels. Massachusetts Institute of Technology.

[5] http://www.makeitfrom.com/compare/7075-T6-Aluminum/ASTM-A36-SS400-S27 5-Structural-Carbon-Steel

[6] https://www.bangslabs.com/sites/default/files/imce/docs/TSD\%200021\%20Material \%20Properties\%20Web.pdf

[7] Wang, C., Chen, H.-R. and Lei, Z.-K. (2010) Experimental Investigation of Interfacial Fracture Behavior in Foam Core Sandwich Beams with Visco-Elastic Adhesive Interface. Composite Structures, 92, 1085-1091.

https://doi.org/10.1016/j.compstruct.2009.10.011

[8] Jakobsen, J., Andreasen, J.H. and Thomsen, O.T. (2009) Crack Deflection by Core Junctions in Sandwich Structures. Engineering Fracture Mechanics, 76, 2135-2147. https://doi.org/10.1016/j.engfracmech.2009.01.013

[9] Gdoutos, E.E., Daniel, I.M. and Wang, K.-A. (2002) Indentation Failure in Composite Sandwich Structures. Experimental Mechanics, 42, 426-431. https://doi.org/10.1007/BF02412148

[10] Allen, H.G. (1969) Analysis and Design of Structural Sandwich Panels. Pergamon Press, London.

[11] Zenkert, D. (1995) An Introduction to Sandwich Construction. Chameleon, London.

[12] Miravete, A. (1994) Optimisation of Design of Composite Structures. Woodhead Publishing Ltd.

[13] Wang, D. (2009) Impact Behavior and Energy Absorption of Paper Honeycomb 
Sandwich Panels. International Journal of Impact Engineering, 36, 110-114.

[14] Dharmasena, K.P., et al. (2008) Mechanical Response of Metallic Honeycomb Sandwich Panel Structures to High-Intensity Dynamic Loading. International Journal of Impact Engineering, 35, 1063-1074.

[15] Burton, W.S. and Noor, A.K. (1997) Assessment of Continuum Models for Sandwich Panel Honeycomb Cores. Computer Methods in Applied Mechanics and Engineering, 145, 341-360. 